\title{
Existence theorems for two-fluid magnetohydrodynamics
}

Manuel Núñez

Citation: Journal of Mathematical Physics 46, 083101 (2005); doi: 10.1063/1.1985009

View online: https://doi.org/10.1063/1.1985009

View Table of Contents: http://aip.scitation.org/toc/jmp/46/8

Published by the American Institute of Physics

\section{Articles you may be interested in}

General theory of the plasmoid instability

Physics of Plasmas 23, 100702 (2016); 10.1063/1.4964481

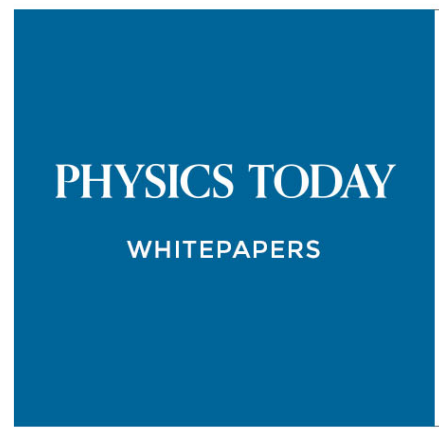

MANAGER'S GUIDE

Accelerate R\&D with

Multiphysics Simulation

\section{READ NOW}

PRESENTED BY $\checkmark \subset \bigcirc M S \bigcirc L$ 


\title{
Existence theorems for two-fluid magnetohydrodynamics
}

\author{
Manuel Núñez ${ }^{\text {a) }}$ \\ Departamento de Análisis Matemático, Universidad de Valladolid, 47005 Valladolid, Spain
}

(Received 5 April 2005; accepted 1 June 2005; published online 27 July 2005)

The description of a plasma as composed by two types of fluids, formed by ions and electrons, is more complete than the classical one-fluid magnetohydrodynamics (MHD) model and it has proved necessary to explain the phenomena of fast magnetic reconnection. We prove a finite-time theorem of existence and uniqueness of solutions for this system for either Dirichlet or periodic boundary conditions in dimension three. It turns out that the regularity estimates for the magnetic field are finer than the MHD ones. (C) 2005 American Institute of Physics.

[DOI: $10.1063 / 1.1985009]$

\section{INTRODUCTION AND MATHEMATICAL SETTING}

The evolution of a collection of charged particles can often be modeled as the motion of several fluid species linked by electromagnetic forces. The most important instance is probably the two-fluid case, where positive ions and electrons form a quasineutral plasma. The equations of motion of both species, under reasonable approximations ${ }^{1-3}$ may be combined to yield

$$
\begin{gathered}
\rho\left(\frac{\partial \mathbf{v}}{\partial t}+\mathbf{v} \cdot \nabla \mathbf{v}\right)=\nu \Delta \mathbf{v}+\mathbf{J} \times \mathbf{B}-\nabla p+\mathbf{f} \\
\mathbf{E}+\mathbf{v} \times \mathbf{B}=\eta \mathbf{J}+\frac{1}{e n} \mathbf{J} \times \mathbf{B}-\frac{1}{e n} \nabla p_{e}+\frac{m}{e^{2} n} \frac{\partial \mathbf{J}}{\partial t},
\end{gathered}
$$

where the variables are explained as follows:

(1) $\mathbf{v}$ is the mass velocity

$$
\mathbf{v}=\frac{M \mathbf{v}_{i}+m \mathbf{v}_{e}}{M+m}
$$

where $\mathbf{v}_{i}$ is the ions velocity, $M$ is its mass, $\mathbf{v}_{e}$ is the electrons velocity, and $m$ is its mass. Since $M \gg m, \mathbf{v} \approx \mathbf{v}_{i}$.

(2) $e$ is the electron charge.

(3) $n$ is the electrons number density, approximately equal to the ions one, $\rho=n(M+m)$ the mass density. We will assume the fluid incompressible and will scale $\rho$ to 1 .

(4) $\nu$ is the kinematic viscosity and $\eta$ is the resistivity. Both are taken as constant (positive) scalars, meaning that these plasma properties are homogeneous and isotropic.

(5) $p=p_{i}+p_{e}$ is the total pressure, sum of the ion and electron ones.

(6) $\mathbf{E}$ is the electric field, $\mathbf{B}$ is the magnetic one, and $\mathbf{J}=\boldsymbol{\nabla} \times \mathbf{B}$ is the current density. This is also equal to $\left(\mathbf{v}_{i}-\mathbf{v}_{e}\right)$ en.

(7) $\mathbf{f}$ is an arbitrary forcing on the momentum equation, given, e.g., by gravitational forces.

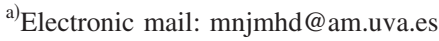


It is apparent that the coefficient $m /\left(e^{2} n\right)$ is very small. Neglecting it we are left with the Hall magnetohydrodynamics (MHD) system. By neglecting also the current displacement in Maxwell's equations, an admissible procedure in low-frequency phenomena, and using Faraday's law

$$
\frac{\partial \mathbf{B}}{\partial t}=-\nabla \times \mathbf{E},
$$

we are left with the Hall induction equation

$$
\frac{\partial \mathbf{B}}{\partial t}=-\nabla \times(\eta \mathbf{J})+\nabla \times(\mathbf{v} \times \mathbf{B})-\frac{1}{e n} \nabla \times(\mathbf{J} \times \mathbf{B}) .
$$

The Hall current term $(1 /$ en $) \nabla \times(\mathbf{J} \times \mathbf{B})$ is small in dense plasmas, and in general at large scales its effect is less important than the one of the velocity. Omitting it and using certain vector analysis identities, we obtain the classical incompressible MHD equations

$$
\begin{gathered}
\frac{\partial \mathbf{v}}{\partial t}=\nu \Delta \mathbf{v}-\mathbf{v} \cdot \nabla \mathbf{v}+\mathbf{B} \cdot \nabla \mathbf{B}-\nabla\left(p+\frac{1}{2} B^{2}\right)+\mathbf{f}, \\
\frac{\partial \mathbf{B}}{\partial t}=\eta \Delta \mathbf{B}+\nabla \times(\mathbf{v} \times \mathbf{B}), \\
\nabla \cdot \mathbf{v}=\nabla \cdot \mathbf{B}=0 .
\end{gathered}
$$

Its study is well known and similar in many aspects to the one of the Navier-Stokes equation. ${ }^{4}$ However, it has become apparent that in certain important physical phenomena, in particular those involving magnetic reconnection, the one-fluid description given by (3) is not appropriate. The extremely rapid conversion rate from magnetic to kinetic energy present in such spectacular phenomena as solar flares could not be adequately explained by any MHD model, and there was no lack of effort in this sense. There exists an enormous literature on this problem: see, e.g., Refs. $5-7$, and references therein. It is generally accepted now that near the current sheets where reconnection occurs, electrons and ions cease to move together: it is the high electron velocity which prevents the throttling of MHD reconnection schemes and allows fast energy conversion. It is clear that a two-fluid description is necessary and therefore it seems natural to ask for some existence theorem for the relevant equation. Although admittedly the electron inertia term $m /\left(e^{2} n\right)$ is small, its presence is necessary to obtain a mathematically consistent model. By taking the curl of (1) and using Faraday's law, we obtain the two-fluid MHD system

$$
\begin{gathered}
\frac{\partial \mathbf{v}}{\partial t}=\nu \Delta \mathbf{v}-\mathbf{v} \cdot \nabla \mathbf{v}+\mathbf{B} \cdot \nabla \mathbf{B}-\nabla\left(p+\frac{1}{2} B^{2}\right)+\mathbf{f}, \\
\frac{m}{e^{2} n} \frac{\partial \nabla \times \mathbf{J}}{\partial t}+\frac{\partial \mathbf{B}}{\partial t}=\eta \Delta \mathbf{B}+\nabla \times(\mathbf{v} \times \mathbf{B})-\frac{1}{e n} \nabla \times(\mathbf{J} \times \mathbf{B}),
\end{gathered}
$$

which will be our object of study. We will abbreviate the electron inertia term $m /\left(e^{2} n\right)$ to $\alpha$, the Hall coefficient $1 /($ en $)$ to $h$. In addition to these equations, both $\mathbf{v}$ and $\mathbf{B}$ must be solenoidal. This follows automatically for $\mathbf{B}$ for all time if it happens for the initial condition at time zero, because the divergence of the induction equation yields 


$$
\frac{\partial(\boldsymbol{\nabla} \cdot \mathbf{B})}{\partial t}=0 .
$$

As for the momentum one, the task of keeping $\mathbf{v}$ solenoidal is given to the pressure: $p$ satisfies an elliptic equation to this end. Indeed, physically the pressure compensates for the tensions created by the fluid incompressibility.

Finally, boundary conditions must be added. Although other possibilities exist, we will concentrate for simplicity on two cases:

(1) The domain $\Omega$ is smooth, and both $\mathbf{v}$ and $\mathbf{B}$ satisfy homogeneous Dirichlet conditions at its boundary $\partial \Omega$.

(2) $\Omega$ is a box $\left(0, L_{1}\right) \times\left(0, L_{2}\right) \times\left(0, L_{3}\right)$, and both $\mathbf{v}$ and $\mathbf{B}$ are periodic at opposite faces. Moreover, in this case the mean of both in $\Omega$ is zero.

Incidently, notice that, in contrast to the MHD and Navier-Stokes cases, there is no possibility here of studying two-dimensional problems, because even when both velocity and magnetic field are horizontal, the current density and the Hall term are not.

We first recall the definitions of the classical Lebesgue and Sobolev spaces: $L^{p}(\Omega)$ is the space of measurable functions $f$ defined in $\Omega$ whose $L^{p}$-norm

$$
\|f\|_{p}=\left(\int_{\Omega}|f|^{p} \mathrm{~d} V\right)^{1 / p}
$$

is bounded. $L^{\infty}(\Omega)$ is the space of measurable functions defined in $\Omega$ such that they are bounded outside a set of zero measure $E$, and $\|f\|_{\infty}$ is the maximum of $|f(\mathbf{x})|$ when $\mathbf{x} \notin E$. $H^{p}(\Omega)$ is the space of functions whose differentials up to the order $p$ are square integrable [i.e., they belong to $L^{2}(\Omega)$ ]. The norm $\|f\|_{H^{p}(\Omega)}$ is the sum of the $L^{2}$-norms of all the partial derivatives of $f$ up to the order $p$. We will only use $H^{1}(\Omega)$ and $H^{2}(\Omega) . H_{0}^{1}(\Omega)$ is the subspace of $H^{1}(\Omega)$ formed (roughly) by the functions vanishing in the boundary $\partial \Omega$. (The correct definition is somewhat more involved, but essentially amounts to this). We will use a number of standard notations: ${ }^{4,8}$

In the Dirichlet case,

$$
H=\left\{\mathbf{B} \in L^{2}(\Omega)^{3}: \boldsymbol{\nabla} \cdot \mathbf{B}=0,\left.\mathbf{B} \cdot \mathbf{n}\right|_{\partial \Omega}=0\right\} .
$$

Although this divergence and trace term must be understood in the sense of distributions, it is known that $H$ is a closed subspace of $L^{2}(\Omega)^{3}$.

$$
\begin{gathered}
V=H_{0}^{1}(\Omega)^{3} \cap H, \\
D(A)=H^{2}(\Omega)^{3} \cap V, \\
A: D(A) \rightarrow H, \quad A=P_{H} \Delta,
\end{gathered}
$$

where $P_{H}$ is the orthogonal projection on $H$. In the periodic case,

$$
H=\left\{\mathbf{u} \in L^{2}(\Omega)^{3}: \int_{\Omega} \mathbf{u} \mathrm{d} V=\mathbf{0}, \quad \boldsymbol{\nabla} \cdot \mathbf{u}=0, \mathbf{u} \cdot \mathbf{n} \text { antiperiodic at opposite sides of } \Omega\right\} .
$$

Again $H$ is a closed subspace of $L^{2}(\Omega)^{3}$

$$
\begin{gathered}
V=H^{1}(\Omega)^{3} \cap H, \\
D(A)=H^{2}(\Omega)^{3} \cap V,
\end{gathered}
$$




$$
A: D(A) \rightarrow H, \quad A=\Delta .
$$

In connection with these spaces, we will use the following inequality (see Ref. 8): For all $\mathbf{f}$ $\in V, \mathbf{g} \in D(A), \mathbf{h} \in H$,

$$
\left|\int_{\Omega} \mathbf{f} \cdot \boldsymbol{\nabla} \mathbf{g} \cdot \mathbf{h} \mathrm{d} V\right| \leqslant c\|\mathbf{f}\|_{H^{1}}\|\mathbf{g}\|_{H^{1}}^{1 / 2}\|A \mathbf{g}\|_{2}^{1 / 2}\|h\|_{2} .
$$

$c$ is a constant depending only on $\Omega$. To avoid an unmanageable proliferation of constants, we will always denote such parameters by $c$ : thus, e.g., $3 c+c^{1 / 2}=c$. Constants not depending on $\Omega$, such as the diffusivity terms or bounds depending on specific functions will be given different names.

We intend to prove local existence and uniqueness of the solution to the problem (4) and (5) with initial condition $\mathbf{v}(0) \in V, \mathbf{B}(0) \in D(A)$. We will consider first the induction equation, which in the MHD case models the so-called kinematic dynamo, where the velocity is taken as a datum. The momentum equation in a classical Navier-Stokes one with a forcing given by the Lorentz force, for which well-known estimates are available. Combining the bounds for both equations we will obtain a contractive operator whose fixed point is the unique solution. Finally we will obtain some energy inequalities emphasizing the difference with the single-fluid MHD model. All these results will hold even in infinitely conducting plasmas $(\eta=0)$, in contrast with classical MHD.

\section{INDUCTION EQUATION}

Definition 2.1: For $\mathbf{B} \in D(A)$ we will denote by $\mathbf{w}$ the function

$$
\mathbf{w}=-\alpha \Delta \mathbf{B}+\mathbf{B} .
$$

Notice that the properties of elliptic systems guarantee that $\mathbf{w} \in H$ and $\|\mathbf{B}\|_{H^{2}} \leqslant c\|\mathbf{w}\|_{2}$.

Lemma 2.2: Consider the term $\eta \Delta \mathbf{B}+\nabla \times(\mathbf{v} \times \mathbf{B})-h \nabla \times(\mathbf{J} \times \mathbf{B})$ as a (nonlocal) function $F(\mathbf{w}, \mathbf{v})$. Then, for every $\mathbf{v} \in V, F$ is a continuous function from $H$ into $H$. Moreover, for any $\mathbf{v}$ $\in V, \mathbf{w} \in H$,

$$
\|F(\mathbf{w}, \mathbf{v})\|_{2} \leqslant c\left(\eta+\|\mathbf{v}\|_{H^{1}}+\|\mathbf{w}\|_{2}\right)\|\mathbf{w}\|_{2},
$$

and for any $\mathbf{v}_{1}, \mathbf{v}_{2} \in V, \mathbf{w}_{1}, \mathbf{w}_{2} \in H$,

$$
\begin{aligned}
& \left\|F\left(\mathbf{w}_{1}, \mathbf{v}_{1}\right)-F\left(\mathbf{w}_{2}, \mathbf{v}_{2}\right)\right\|_{2} \\
& \quad \leqslant c\left(\eta+\left\|\mathbf{v}_{1}\right\|_{H^{1}}+\left\|\mathbf{v}_{2}\right\|_{H^{1}}+\left\|\mathbf{w}_{1}\right\|_{2}+\left\|\mathbf{w}_{2}\right\|_{2}\right)\left\|\mathbf{w}_{1}-\mathbf{w}_{2}\right\|_{2}+c\left(\left\|\mathbf{w}_{1}\right\|_{2}+\left\|\mathbf{w}_{2}\right\|\right)\left\|\mathbf{v}_{1}-\mathbf{v}_{2}\right\|_{H^{1}} .
\end{aligned}
$$

Proof: Recall that

$$
\begin{aligned}
& \nabla \times(\mathbf{v} \times \mathbf{B})=-\mathbf{v} \cdot \nabla \mathbf{B}+\mathbf{B} \cdot \nabla \mathbf{v}, \\
& \nabla \times(\mathbf{J} \times \mathbf{B})=-\mathbf{J} \cdot \nabla \mathbf{B}+\mathbf{B} \cdot \nabla \mathbf{J} .
\end{aligned}
$$

By standard Sobolev inequalities:

$$
\eta\left\|\Delta \mathbf{B}_{1}-\Delta \mathbf{B}_{2}\right\|_{2} \leqslant c \eta\left\|\mathbf{B}_{1}-\mathbf{B}_{2}\right\|_{H^{2}} \leqslant c \eta\left\|\mathbf{w}_{1}-\mathbf{w}_{2}\right\|_{2},
$$

and

$$
\begin{aligned}
\| \nabla \times & \left(\mathbf{v}_{1} \times \mathbf{B}_{1}\right)-\nabla \times\left(\mathbf{v}_{2} \times \mathbf{B}_{2}\right) \|_{2} \\
& =\left\|-\mathbf{v}_{1} \cdot \boldsymbol{\nabla}\left(\mathbf{B}_{1}-\mathbf{B}_{2}\right)-\left(\mathbf{v}_{1}-\mathbf{v}_{2}\right) \cdot \boldsymbol{\nabla} \mathbf{B}_{2}+\mathbf{B}_{1} \cdot \boldsymbol{\nabla}\left(\mathbf{v}_{1}-\mathbf{v}_{2}\right)+\left(\mathbf{B}_{1}-\mathbf{B}_{2}\right) \cdot \boldsymbol{\nabla} \mathbf{v}_{2}\right\|_{2} \\
& \leqslant\left\|\mathbf{v}_{1}\right\|_{4}\left\|\boldsymbol{\nabla}\left(\mathbf{B}_{1}-\mathbf{B}_{2}\right)\right\|_{4}+\left\|\mathbf{v}_{1}-\mathbf{v}_{2}\right\|_{4}\left\|\boldsymbol{\nabla} \mathbf{B}_{2}\right\|_{4}+\left\|\mathbf{B}_{1}\right\|_{\infty}\left\|\boldsymbol{\nabla}\left(\mathbf{v}_{1}-\mathbf{v}_{2}\right)\right\|_{2}+\left\|\mathbf{B}_{1}-\mathbf{B}_{2}\right\|_{\infty}\left\|\boldsymbol{\nabla} \mathbf{v}_{2}\right\|_{2} .
\end{aligned}
$$

Since for dimension 3, $V \subset L^{4}(\Omega), D(A) \subset L^{\infty}(\Omega)$, this amount may be bounded by 


$$
\begin{aligned}
& c\left(\left\|\mathbf{v}_{1}\right\|_{H^{1}}\left\|\mathbf{B}_{1}-\mathbf{B}_{2}\right\|_{H^{2}}+\left\|\mathbf{B}_{2}\right\|_{H^{2}}\left\|\mathbf{v}_{1}-\mathbf{v}_{2}\right\|_{H^{1}}+\left\|\mathbf{B}_{1}\right\|_{H^{2}}\left\|\mathbf{v}_{1}-\mathbf{v}_{2}\right\|_{H^{1}}+\left\|\mathbf{v}_{2}\right\|\left\|_{H^{1}}\right\| \mathbf{B}_{1}-\mathbf{B}_{2} \|_{H^{2}}\right. \\
& \quad \leqslant c\left(\left\|\mathbf{v}_{1}\right\|_{H^{1}}+\left\|\mathbf{v}_{2}\right\|_{H^{1}}\right)\left\|\mathbf{w}_{1}-\mathbf{w}_{2}\right\|_{2}+c\left(\left\|\mathbf{w}_{1}\right\|_{2}+\|\mathbf{w}\|_{2}\right)\left\|\mathbf{v}_{1}-\mathbf{v}_{2}\right\|_{H^{1}} .
\end{aligned}
$$

Finally, by changing in the previous inequalities $\mathbf{v}_{i}$ to $\mathbf{J}_{i}$ we find

$\left\|\nabla \times\left(\mathbf{J}_{1} \times \mathbf{B}_{1}\right)-\nabla \times\left(\mathbf{J}_{2} \times \mathbf{B}_{2}\right)\right\|_{2}$

$$
\begin{aligned}
& \leqslant\left\|\mathbf{J}_{1}\right\|_{4}\left\|\nabla\left(\mathbf{B}_{1}-\mathbf{B}_{2}\right)\right\|_{4}+\left\|\mathbf{J}_{1}-\mathbf{J}_{2}\right\|_{4}\left\|\boldsymbol{\nabla} \mathbf{B}_{2}\right\|_{4}+\left\|\mathbf{B}_{1}\right\|_{\infty}\left\|\nabla\left(\mathbf{J}_{1}-\mathbf{J}_{2}\right)\right\|_{2}+\left\|\mathbf{B}_{1}-\mathbf{B}_{2}\right\|_{\infty}\left\|_{\boldsymbol{~}} \mathbf{J}_{2}\right\|_{2} \\
& \leqslant c\left(\left\|\mathbf{J}_{1}\right\|_{H^{1}}+\left\|\mathbf{B}_{2}\right\|_{H^{2}}+\left\|\mathbf{B}_{1}\right\|_{H^{2}}+\left\|\mathbf{J}_{2}\right\|_{H^{1}}\right)\left\|\mathbf{B}_{1}-\mathbf{B}_{2}\right\|_{H^{2}} \leqslant c\left(\left\|\mathbf{w}_{1}\right\|_{2}+\left\|\mathbf{w}_{2}\right\|_{2}\right)\left\|\mathbf{w}_{1}-\mathbf{w}_{2}\right\|_{2} .
\end{aligned}
$$

The inequality (12) is a simplified version of the previous calculation, with only a single $\mathbf{v}$ and $\mathbf{w}$. This concludes the proof.

Theorem 2.3: For fixed $\mathbf{v} \in L^{2}((0, S), V)$, Eq. (5), which may be written in the form

$$
\begin{gathered}
\frac{\mathrm{d} \mathbf{w}}{\mathrm{d} t}=F(\mathbf{w}, \mathbf{v}), \\
\mathbf{w}(0) \in H,
\end{gathered}
$$

admits a unique a.e. differentiable solution $\mathbf{w}:(0, T) \rightarrow H$, for some $T \leqslant S, T>0$. We may take $T$ so that this solution satisfies $\|\mathbf{w}(t)\|_{2} \leqslant\|\mathbf{w}(0)\|_{2}+1$ for all $t \in(0, T)$. Moreover, the interval $(0, T)$ depends only on $\|\mathbf{w}(0)\|_{2}$ and $\|\mathbf{v}\|_{L^{2}((0, T), V)}$.

Proof: Let us prove that the integral operator, defined in the closed ball of $\mathcal{C}((0, T), H)$ of center $\mathbf{0}$ and radius $\|\mathbf{w}(0)\|_{2}+1$ given by

$$
\mathbf{w} \rightarrow \mathbf{w}(0)+\int_{0}^{t} F(\mathbf{w}(s), \mathbf{v}(s)) \mathrm{d} s,
$$

takes the ball to itself and is contractive for $T$ small enough. Using (12), for any $\mathbf{w}$ bounded by $\|\mathbf{w}(0)\|_{2}+1$,

$$
\begin{aligned}
\int_{0}^{t}\|F(\mathbf{w}, \mathbf{v})\|_{2} \mathrm{~d} s & \leqslant c \int_{0}^{t}\left(\eta+\|\mathbf{v}\|_{H^{1}}+\|\mathbf{w}\|_{2}\right)\|\mathbf{w}\|_{2} \mathrm{~d} s \\
& \leqslant c\left(\sqrt{T}\left(\int_{0}^{T}\|\mathbf{v}\|_{H^{1}}^{2} \mathrm{~d} s\right)^{1 / 2}+T\left(\eta+\|\mathbf{w}(0)\|_{2}+1\right)\right)\left(\|\mathbf{w}(0)\|_{2}+1\right) .
\end{aligned}
$$

Clearly we can take $T$ so that this amount is less than 1 for all $t \in(0, T)$. Taking now (13),

$$
\int_{0}^{t}\left\|F\left(\mathbf{w}_{1}, \mathbf{v}\right)-F\left(\mathbf{w}_{2}, \mathbf{v}\right)\right\|_{2} \mathrm{~d} s \leqslant c \int_{0}^{t}\left(\eta+2\|\mathbf{v}\|_{H^{1}}+\left\|\mathbf{w}_{1}\right\|_{2}+\left\|\mathbf{w}_{2}\right\|_{2}\right)\left\|\mathbf{w}_{1}-\mathbf{w}_{2}\right\|_{2} \mathrm{~d} s,
$$

so that

$$
\begin{aligned}
\left\|\mathbf{w}_{1}-\mathbf{w}_{2}\right\|_{\mathcal{C}(0, T), H} & \leqslant c\left(\int_{0}^{T} 2\|v\|_{H^{1}}+\left\|\mathbf{w}_{1}\right\|_{2}+\left\|\mathbf{w}_{2}\right\|_{2} \mathrm{~d} s\right)\left\|\mathbf{w}_{1}-\mathbf{w}_{2}\right\|_{\mathcal{C}(0, T), H} \\
& \leqslant c\left(2 \sqrt{T}\left(\int_{0}^{T}\|\mathbf{v}\|_{H^{1}}^{2} \mathrm{~d} s\right)^{1 / 2}+2 T\left(\|\mathbf{w}(0)\|_{2}+1\right)\right)\left\|\mathbf{w}_{1}-\mathbf{w}_{2}\right\|_{\mathcal{C}(0, T), H} .
\end{aligned}
$$

Obviously by taking $T$ small enough we can make this functional to take the ball of radius $\|\mathbf{w}(0)\|_{2}+1$ in itself in a contractive manner. The classical proof follows.

Let us study now the dependence of the solutions upon the velocity:

Theorem 2.4: Let $\mathbf{w}_{1}, \mathbf{w}_{2}$ be the respective solutions of 


$$
\begin{gathered}
\frac{\mathrm{d} \mathbf{w}_{i}}{\mathrm{~d} t}=F\left(\mathbf{w}_{i}, \mathbf{v}_{i}\right) \\
\mathbf{w}_{i}(0)=\mathbf{w}(0),
\end{gathered}
$$

for $\mathbf{v}_{i} \in L^{2}((0, S), V)$, and let $(0, T)$ be a common interval of existence of $\mathbf{w}_{1}, \mathbf{w}_{2}$. Then there exists $T_{1} \leqslant T$, depending only on $\|\mathbf{w}(0)\|_{2},\left\|\mathbf{v}_{i}\right\|_{L^{2}((0, T), V)}$, such that

$$
\left\|\mathbf{w}_{1}-\mathbf{w}_{2}\right\|_{\mathcal{C}\left(\left(0, T_{1}\right), H\right)} \leqslant \frac{1}{2}\left\|\mathbf{v}_{1}-\mathbf{v}_{2}\right\|_{L^{2}\left(\left(0, T_{1}\right), V\right)} .
$$

Proof: Using inequality (13), and taking an interval short enough for Therom 2.3 to hold for both $\mathbf{w}_{i}$,

$$
\begin{aligned}
\left\|\mathbf{w}_{1}(t)-\mathbf{w}_{2}(t)\right\|_{2}= & \left\|\int_{0}^{t} F\left(\mathbf{w}_{1}, \mathbf{v}_{1}\right)-F\left(\mathbf{w}_{2}, \mathbf{v}_{2}\right) \mathrm{d} s\right\| \leqslant \int_{2}^{t}\left(\eta+\left\|\mathbf{v}_{1}\right\|_{H^{1}}+\left\|\mathbf{v}_{2}\right\|_{H^{1}}+\left\|\mathbf{w}_{1}\right\|_{2}+\left\|\mathbf{w}_{2}\right\|_{2}\right) \| \mathbf{w}_{1} \\
& -\mathbf{w}_{2}\left\|_{2}+\left(\left\|\mathbf{w}_{1}\right\|_{2}+\left\|\mathbf{w}_{2}\right\|_{2}\right)\right\|_{\mathbf{v}_{1}}-\mathbf{v}_{2} \|_{H^{1}} \mathrm{~d} s \leqslant c\left[\sqrt{T}\left(\left\|\mathbf{v}_{1}\right\|_{L^{2}((0, T), V)}+\left\|\mathbf{v}_{2}\right\|_{L^{2}((0, T), V)}\right)+T \eta\right. \\
& \left.+2 T\left(\|\mathbf{w}(0)\|_{2}+1\right)\right]\left\|\mathbf{w}_{1}-\mathbf{w}_{2}\right\|_{\mathcal{C}((0, T), H)}+2 \sqrt{T}\left(\|\mathbf{w}(0)\|_{2}+1\right)\left\|\mathbf{v}_{1}-\mathbf{v}_{2}\right\|_{L^{2}((0, T), V)} .
\end{aligned}
$$

Hence it is enough to take $T_{1}$ small enough for

$$
c\left[\sqrt{T_{1}}\left(\left\|\mathbf{v}_{1}\right\|_{L^{2}\left(\left(0, T_{1}\right), V\right)}+\left\|\mathbf{v}_{2}\right\|_{L^{2}\left(\left(0, T_{1}\right), V\right)}\right)+T_{1} \eta+2 T_{1}\left(\|\mathbf{w}(0)\|_{2}+1\right)\right]<\frac{1}{2}, 2 \sqrt{T_{1}}\left(\|\mathbf{w}(0)\|_{2}+1\right)<\frac{1}{4} .
$$

\section{MOMENTUM EQUATION}

This is the Navier-Stokes one with two forcings: the independent one $\mathbf{f}$ and the Lorentz force $\mathbf{J} \times \mathbf{B}$. We will denote the last one by $G(\mathbf{w})$. Notice that

$$
\|\mathbf{J} \times \mathbf{B}\|_{2} \leqslant\|\mathbf{J}\|_{4}\|\mathbf{B}\|_{4} \leqslant c\|\mathbf{w}\|_{2}^{2} .
$$

We may apply the classical results (see, e.g., Ref. 8) and conclude the following.

(a) For every initial condition $\mathbf{v}(0) \in V$, there exists a unique solution for an interval $(0, T)$ satisfying

$$
\|\mathbf{v}(t)\|_{H^{1}} \leqslant 2\left(\|\mathbf{v}(0)\|_{H^{1}}+1\right),
$$

where $T$ depends only on $\|\mathbf{v}(0)\|_{H^{1}},\|G(\mathbf{w})\|_{\mathcal{C}((0, T), H)}, \sup _{(0, T)}\|\mathbf{f}\|_{2}$.

(b) $\mathbf{v} \in L^{2}((0, T), D(A))$, and the norm of $\mathbf{v}$ in this space is bounded by a constant depending only on the same quantities given in (a). We will first estimate the norm of $\mathbf{v}$ in the space $L^{2}((0, T), V)$. To emphasize its value, it is convenient to single out (only in this instance) the Poincaré constant in $V,\|\mathbf{v}\|_{H^{1}}^{2} \leqslant k_{p}\|\nabla \mathbf{v}\|_{2}^{2}$.

Proposition 3.1.: If $\mathbf{v}$ is the solution of (4) in the interval $(0, T)$,

$$
\|\mathbf{v}\|_{L^{2}((0, T), V)}^{2} \leqslant \frac{k_{p}}{\nu}\|\mathbf{v}(0)\|_{2}^{2}+c \frac{T}{\nu^{2}}\|\mathbf{w}\|_{\mathcal{C}((0, T), H)}^{4}+c \frac{T}{\nu^{2}} \sup _{(0, T)}\|\mathbf{f}\|_{2}^{2} .
$$

Proof: By making the scalar product of (4) with $\mathbf{v}$, and using standard inequalities (CauchySchwarz, Poincaré, and Young's), 


$$
\begin{aligned}
\frac{1}{2} \frac{d\|\mathbf{v}\|_{2}^{2}}{d t} & \leqslant-\nu\|\nabla \mathbf{v}\|_{2}^{2}+\|G(\mathbf{w})\|_{2}\|\mathbf{v}\|_{2}+\|\mathbf{f}\|_{2}\|\mathbf{v}\|_{2} \leqslant-\nu\|\nabla \mathbf{v}\|_{2}^{2}+c\|G(\mathbf{w})\|_{2}\|\nabla \mathbf{v}\|_{2}+c\|\mathbf{f}\|_{2}\|\nabla \mathbf{v}\|_{2} \\
& \leqslant-\nu\|\nabla \mathbf{v}\|_{2}^{2}+\frac{c^{2}}{\nu}\|G(\mathbf{w})\|_{2}^{2}+\frac{\nu}{4}\|\nabla \mathbf{v}\|_{2}^{2}+\frac{c^{2}}{\nu}\|\mathbf{f}\|_{2}^{2}+\frac{\nu}{4}\|\nabla \mathbf{v}\|_{2}^{2} .
\end{aligned}
$$

Integrating in $(0, T)$ and omitting the term in $\|\mathbf{v}(t)\|_{2}$,

$$
\frac{\nu}{2} \int_{0}^{T}\|\nabla \mathbf{v}\|_{2}^{2} \mathrm{~d} s \leqslant \frac{1}{2}\|\mathbf{v}(0)\|_{2}^{2}+\frac{c^{2}}{\nu} \int_{0}^{T}\|G(\mathbf{w})\|_{2}^{2} \mathrm{~d} s+\frac{c^{2}}{\nu} \int_{0}^{T}\|\mathbf{f}\|_{2}^{2} \mathrm{~d} s,
$$

from which, in view of (17), the result follows.

Corollary 3.2.: There exists a time $T_{1} \leqslant T$, depending only on $\|\mathbf{w}\|_{\mathcal{C}\left(\left(0, T_{1}\right), H\right)}$, such that

$$
\|\mathbf{v}\|_{L^{2}\left(\left(0, T_{1}\right), V\right)} \leqslant \frac{k_{p}}{\sqrt{\nu}}\|\mathbf{v}(0)\|_{2}+1 \leqslant \frac{k_{p}}{\sqrt{\nu}}\|\mathbf{v}(0)\|_{H^{1}}+1 .
$$

We will consider now the difference between two solutions corresponding to different $\mathbf{w}_{i}$ and the same initial condition:

Theorem 3.2: Let $\mathbf{v}_{1}, \mathbf{v}_{2}$ be the respective solutions of Eq. (4) in $(0, T)$ corresponding to Lorentz forces $\mathbf{w}_{1}, \mathbf{w}_{2} \in \mathcal{C}((0, T), H)$ and the same initial condition. Then there exists $T_{2} \leqslant T$, depending only on $\left\|\mathbf{w}_{i}\right\|_{\mathcal{C}((0, T), H)}$, such that

$$
\left\|\mathbf{v}_{1}-\mathbf{v}_{2}\right\|_{L^{2}\left(\left(0, T_{2}\right), V\right)} \leqslant \frac{1}{2}\left\|\mathbf{w}_{1}-\mathbf{w}_{2}\right\|_{\mathcal{C}\left(\left(0, T_{2}\right), H\right)} .
$$

Proof: The scalar product of $\left(\partial\left(\mathbf{v}_{1}-\mathbf{v}_{2}\right)\right) /(\partial t)$ with $\mathbf{v}_{1}-\mathbf{v}_{2}$ yields

$$
\begin{aligned}
\frac{1}{2} \frac{\partial}{\partial t}\left\|\mathbf{v}_{1}-\mathbf{v}_{2}\right\|_{2}^{2}+\nu\left\|\nabla\left(\mathbf{v}_{1}-\mathbf{v}_{2}\right)\right\|_{2}^{2}= & \left(-\mathbf{v}_{1} \cdot \nabla \mathbf{v}_{1}+\mathbf{v}_{2} \cdot \nabla \mathbf{v}_{2}, \mathbf{v}_{1}-\mathbf{v}_{2}\right)+\left(G\left(\mathbf{w}_{1}\right)-G\left(\mathbf{w}_{2}\right), \mathbf{v}_{1}-\mathbf{v}_{2}\right) \\
& -\left(\nabla\left(p_{1}-p_{2}\right), \mathbf{v}_{1}-\mathbf{v}_{2}\right) .
\end{aligned}
$$

The last term is zero. We have

$$
\left(-\mathbf{v}_{1} \cdot \nabla \mathbf{v}_{1}+\mathbf{v}_{2} \cdot \nabla \mathbf{v}_{2}, \mathbf{v}_{1}-\mathbf{v}_{2}\right)=\left(-\mathbf{v}_{1} \cdot \nabla\left(\mathbf{v}_{1}-\mathbf{v}_{2}\right)+\left(-\mathbf{v}_{1}+\mathbf{v}_{2}\right) \cdot \nabla \mathbf{v}_{2}, \mathbf{v}_{1}-\mathbf{v}_{2}\right),
$$

and $\left(-\mathbf{v}_{1} \cdot \nabla\left(\mathbf{v}_{1}-\mathbf{v}_{2}\right), \mathbf{v}_{1}-\mathbf{v}_{2}\right)=0$. By using inequality (10), we find

$$
\left|\left(-\mathbf{v}_{1} \cdot \nabla \mathbf{v}_{1}+\mathbf{v}_{2} \cdot \nabla \mathbf{v}_{2}, \mathbf{v}_{1}-\mathbf{v}_{2}\right)\right| \leqslant\left\|\mathbf{v}_{1}-\mathbf{v}_{2}\right\|_{H^{1}}\left\|\mathbf{v}_{1}-\mathbf{v}_{2}\right\|\left\|_{2}\right\| \mathbf{v}_{2}\left\|_{H^{1}}^{1 / 2}\right\| A \mathbf{v}_{2} \|_{2}^{1 / 2}
$$

Finally

$$
\left|\left(G\left(\mathbf{w}_{1}\right)-G\left(\mathbf{w}_{2}\right), \mathbf{v}_{1}-\mathbf{v}_{2}\right)\right| \leqslant\left\|G\left(\mathbf{w}_{1}\right)-G\left(\mathbf{w}_{2}\right)\right\|_{2}\left\|\mathbf{v}_{1}-\mathbf{v}_{2}\right\|_{2} .
$$

In order to abbreviate the notation, let us denote by $\|g\|_{2, \infty}$ the supremum of $\|g\|_{2}$ when $t \in(0, T)$. Property (a) of the solutions of the Navier-Stokes equations guarantees that $\left\|\mathbf{v}_{2}\right\|_{H^{1}}$ is bounded in $(0, T)$ by an amount depending only on $\left\|\mathbf{v}_{2}(0)\right\|_{H^{1}},\left\|G\left(\mathbf{w}_{2}\right)\right\|_{2, \infty}$, and $\|\mathbf{f}\|_{2, \infty}$. Let $M$ be an upper bound of $\left\|\mathbf{v}_{2}\right\|_{H^{1}}^{1 / 2}$ in $(0, T)$. Integrating the resulting inequality in time, 


$$
\begin{aligned}
& \frac{1}{2}\left\|\left(\mathbf{v}_{1}-\mathbf{v}_{2}\right)(t)\right\|_{2}^{2}+\nu \int_{0}^{t}\left\|\nabla\left(\mathbf{v}_{1}-\mathbf{v}_{2}\right)\right\|_{2}^{2} \mathrm{~d} s \\
& \leqslant c M \int_{0}^{t}\left\|\mathbf{v}_{1}-\mathbf{v}_{2}\right\|_{2}\left\|\mathbf{v}_{1}-\mathbf{v}_{2}\right\|_{H^{1}}\left\|\mathbf{v}_{2}\right\|_{H^{2}}^{1 / 2} \mathrm{~d} s+c \int_{0}^{t}\left\|G\left(\mathbf{w}_{1}\right)-G\left(\mathbf{w}_{2}\right)\right\|_{2}\left\|\mathbf{v}_{1}-\mathbf{v}_{2}\right\|_{2} \mathrm{~d} s \\
& \leqslant c M\left\|\mathbf{v}_{1}-\mathbf{v}_{2}\right\|_{2, \infty}\left(\int_{0}^{T}\left\|\mathbf{v}_{1}-\mathbf{v}_{2}\right\|_{H^{1}}^{2} \mathrm{~d} s\right)^{1 / 2}\left(\int_{0}^{T}\left\|\mathbf{v}_{2}\right\|_{H^{2}} \mathrm{~d} s\right)^{1 / 2} \\
& +c T\left\|G\left(\mathbf{w}_{1}\right)-G\left(\mathbf{w}_{2}\right)\right\|_{2, \infty}\left\|\mathbf{v}_{1}-\mathbf{v}_{2}\right\|_{2, \infty} .
\end{aligned}
$$

Since this is valid for any $t \in(0, T)$, by considering only the first term in the left-hand side of (22) and using Young's inequality,

$$
\begin{aligned}
\frac{1}{2}\left\|\mathbf{v}_{1}-\mathbf{v}_{2}\right\|_{2, \infty}^{2} \leqslant & \frac{c^{2} M^{2}}{2}\left(\int_{0}^{T}\left\|\mathbf{v}_{2}\right\|_{H^{2}} \mathrm{~d} s\right)\left\|\mathbf{v}_{1}-\mathbf{v}_{2}\right\|_{2, \infty}^{2}+\frac{1}{2} \int_{0}^{T}\left\|\mathbf{v}_{1}-\mathbf{v}_{2}\right\|_{H^{1}}^{2} \mathrm{~d} s+\frac{T}{2}\left\|G\left(\mathbf{w}_{1}\right)-G\left(\mathbf{w}_{2}\right)\right\|_{2, \infty}^{2} \\
& +\frac{c^{2}}{2} T\left\|\mathbf{v}_{1}-\mathbf{v}_{2}\right\|_{2, \infty}^{2} .
\end{aligned}
$$

Since by property (b)

$$
\int_{0}^{T}\left\|\mathbf{v}_{2}\right\|_{H^{2}} \mathrm{~d} s \leqslant \sqrt{T}\left(\int_{0}^{T}\left\|\mathbf{v}_{2}\right\|_{H^{2}}^{2} \mathrm{~d} s\right)^{1 / 2} \leqslant N \sqrt{T}
$$

where $N$ is a constant depending on the same functions stated before for $\left\|\mathbf{v}_{2}\right\|_{H^{1}}$, we find

$$
\left(1-c^{2} M^{2} N \sqrt{T}-c^{2} T\right)\left\|\mathbf{v}_{1}-\mathbf{v}_{2}\right\|_{2, \infty}^{2} \leqslant \int_{0}^{T}\left\|\mathbf{v}_{1}-\mathbf{v}_{2}\right\|_{H^{1}}^{2} \mathrm{~d} s+T\left\|G\left(\mathbf{w}_{1}\right)-G\left(\mathbf{w}_{2}\right)\right\|_{2, \infty}^{2}
$$

Taking $T$ small enough for the constant before $\left\|\mathbf{v}_{1}-\mathbf{v}_{2}\right\|_{2, \infty}^{2}$ to be at least $1 / 2$, we find

$$
\left\|\mathbf{v}_{1}-\mathbf{v}_{2}\right\|_{2, \infty}^{2} \leqslant 2 \int_{0}^{T}\left\|\mathbf{v}_{1}-\mathbf{v}_{2}\right\|_{H^{1}}^{2} \mathrm{~d} s+2 T\left\|G\left(\mathbf{w}_{1}\right)-G\left(\mathbf{w}_{2}\right)\right\|_{2, \infty}^{2}
$$

which implies

$$
\left\|\mathbf{v}_{1}-\mathbf{v}_{2}\right\|_{2, \infty} \leqslant \sqrt{2}\left(\int_{0}^{T}\left\|\mathbf{v}_{1}-\mathbf{v}_{2}\right\|_{H^{1}}^{2} \mathrm{~d} s\right)^{1 / 2}+\sqrt{2} T\left\|G\left(\mathbf{w}_{1}\right)-G\left(\mathbf{w}_{2}\right)\right\|_{2, \infty} .
$$

Let us now return to (22) omitting now the term $\left\|\left(\mathbf{v}_{1}-\mathbf{v}_{2}\right)(t)\right\|_{2, \infty}^{2}$, and bounding the last term in a slightly different form:

$$
\begin{aligned}
\int_{0}^{T}\left\|G\left(\mathbf{w}_{1}\right)-G\left(\mathbf{w}_{2}\right)\right\|_{2}\left\|\mathbf{v}_{1}-\mathbf{v}_{2}\right\| \mathrm{d} s & \leqslant\left\|G\left(\mathbf{w}_{1}\right)-G\left(\mathbf{w}_{2}\right)\right\|_{2, \infty} \int_{0}^{T}\left\|\mathbf{v}_{1}-\mathbf{v}_{2}\right\|_{2} \mathrm{~d} s \\
& \leqslant\left\|G\left(\mathbf{w}_{1}\right)-G\left(\mathbf{w}_{2}\right)\right\|_{2, \infty} \int_{0}^{T}\left\|\mathbf{v}_{1}-\mathbf{v}_{2}\right\|_{H^{1}} \mathrm{~d} s .
\end{aligned}
$$

By using again Poincaré's inequality on $\mathbf{v}_{1}-\mathbf{v}_{2}$, we get 


$$
\begin{aligned}
& \nu \int_{0}^{T}\left\|\mathbf{v}_{1}-\mathbf{v}_{2}\right\|_{H^{1}}^{2} \mathrm{~d} s \\
& \quad \leqslant c M\left\|\mathbf{v}_{1}-\mathbf{v}_{2}\right\|_{2, \infty}\left(\int_{0}^{T}\left\|\mathbf{v}_{1}-\mathbf{v}_{2}\right\|_{H^{1}}^{2} \mathrm{~d} s\right)^{1 / 2}\left(\int_{0}^{T}\left\|\mathbf{v}_{2}\right\|_{H^{2}} \mathrm{~d} s\right)^{1 / 2} \\
& \quad+c\left\|G\left(\mathbf{w}_{1}\right)-G\left(\mathbf{w}_{2}\right)\right\|_{2, \infty} \int_{0}^{T}\left\|\mathbf{v}_{1}-\mathbf{v}_{2}\right\|_{H^{1}} \mathrm{~d} s .
\end{aligned}
$$

This, jointly with (23), yields

$$
\begin{aligned}
& \nu \int_{0}^{T}\left\|\mathbf{v}_{1}-\mathbf{v}_{2}\right\|_{H^{1}}^{2} \mathrm{~d} s \leqslant c M\left(\int_{0}^{T}\left\|\mathbf{v}_{1}-\mathbf{v}_{2}\right\|_{H^{1}}^{2} \mathrm{~d} s\right)\left(\int_{0}^{T}\left\|\mathbf{v}_{2}\right\|_{H^{2}} \mathrm{~d} s\right)^{1 / 2} \\
& \quad+c M \sqrt{T}\left\|G\left(\mathbf{w}_{1}\right)-G\left(\mathbf{w}_{2}\right)\right\|_{2, \infty}\left(\int_{0}^{T}\left\|\mathbf{v}_{1}-\mathbf{v}_{2}\right\|_{H^{1}}^{2} \mathrm{~d} s\right)^{1 / 2}\left(\int_{0}^{T}\left\|\mathbf{v}_{2}\right\|_{H^{2}} \mathrm{~d} s\right)^{1 / 2} \\
& +c \sqrt{T}\left\|G\left(\mathbf{w}_{1}\right)-G\left(\mathbf{w}_{2}\right)\right\|_{2, \infty}\left(\int_{0}^{T}\left\|\mathbf{v}_{1}-\mathbf{v}_{2}\right\|_{H^{1}}^{2} \mathrm{~d} s\right)^{1 / 2} .
\end{aligned}
$$

Our previous bound on the $H^{2}$ norm of $\mathbf{v}_{2}$ and Young's inequality yield

$$
\begin{aligned}
& \nu \int_{0}^{T}\left\|\mathbf{v}_{1}-\mathbf{v}_{2}\right\|_{H^{1}}^{2} \mathrm{~d} s \leqslant c M N^{1 / 2} T^{1 / 4} \int_{0}^{T}\left\|\mathbf{v}_{1}-\mathbf{v}_{2}\right\|_{H^{1}}^{2} \mathrm{~d} s \\
& \quad+c M N^{1 / 2} T^{3 / 4}\left\|G\left(\mathbf{w}_{1}\right)-G\left(\mathbf{w}_{2}\right)\right\|_{2, \infty}\left(\int_{0}^{T}\left\|\mathbf{v}_{1}-\mathbf{v}_{2}\right\|_{H^{1}}^{2} \mathrm{~d} s\right)^{1 / 2} \\
& \quad+c \sqrt{T}\left\|G\left(\mathbf{w}_{1}\right)-G\left(\mathbf{w}_{2}\right)\right\|_{2, \infty}\left(\int_{0}^{T}\left\|\mathbf{v}_{1}-\mathbf{v}_{2}\right\|_{H^{1}}^{2} \mathrm{~d} s\right)^{1 / 2} \\
& \quad \leqslant\left(c M N^{1 / 2} T^{1 / 4}+\frac{c^{2} M^{2} N}{2} T^{3 / 4}+\frac{c^{2}}{2} T^{1 / 2}\right) \int_{0}^{T}\left\|\mathbf{v}_{1}-\mathbf{v}_{2}\right\|_{H^{1}}^{2} \mathrm{~d} s \\
& \quad+\frac{1}{2}\left(T^{3 / 4}+T^{1 / 2}\right)\left\|G\left(\mathbf{w}_{1}\right)-G\left(\mathbf{w}_{2}\right)\right\|_{2, \infty}^{2} .
\end{aligned}
$$

It is therefore to take $T_{2} \leqslant T$ small enough for

$$
c M N^{1 / 2} T_{2}^{1 / 4}+c^{2} M^{2} N T_{2}^{3 / 4}+c^{2} T_{2}^{1 / 2} \leqslant \frac{\nu}{2}, T_{2}^{3 / 4}+T_{2}^{1 / 2} \leqslant \frac{\nu}{2},
$$

for the theorem to hold. Notice that if we know an a priori bound on

$$
\left\|\mathbf{w}_{i}\right\|_{2, \infty}=\left\|\mathbf{w}_{i}\right\|_{\mathcal{C}((0, T), H)}
$$

(say $\|\mathbf{w}(0)\|_{2}+1$ ), then $M$ and $N$ may also be bounded a priori and $T_{2}$ does not depend on any unknown quantity.

\section{EXISTENCE AND UNIQUENESS OF THE SOLUTIONS}

We now state the main theorem of the paper. For the benefit of readers unwilling to spend time mastering all the previous mathematical notations, perhaps it is appropriate to state the result, at least approximately, in words: When the velocity at time $t=0$ is differentiable, and the magnetic field is twice differentiable, there exists a solution of the two-fluid magnetohydrodynamics equations up to some time T. This solution is such that the velocity and its differential (with respect to 
the space variables) are continuous in time, and the magnetic field, plus its first and second differentials, are also continous in time. The rigorous form is as follows.

Theorem 4.1: Take initial conditions $\mathbf{v}(0)=\mathbf{v}_{0} \in V, \mathbf{B}(0)=\mathbf{B}_{0} \in D(A)$. Then there exists an interval $[0, T]$ such that the two-fluid MHD Eqs. (4) and (5) have a unique solution. Moreover

$$
\begin{gathered}
\mathbf{v} \in \mathcal{C}((0, T), V) \cap L^{2}((0, T), D(A)), \\
\mathbf{B} \in \mathcal{C}((0, T), D(A)) .
\end{gathered}
$$

Proof: Take $T>0$ small enough for:

(a) For $\mathbf{v}^{*} \in L^{2}((0, T), V)$, with

$$
\left\|\mathbf{v}^{*}\right\|_{L^{2}((0, T), V)} \leqslant \frac{k_{p}}{\sqrt{\nu}}\left\|\mathbf{v}_{0}\right\|_{H^{1}}+1
$$

the solution of

$$
\begin{gathered}
\frac{\mathrm{d} \mathbf{w}}{\mathrm{d} t}=F\left(\mathbf{w}, \mathbf{v}^{*}\right), \\
\mathbf{w}(0)=\mathbf{w}_{0}=-\alpha \Delta \mathbf{B}_{0}+\mathbf{B}_{0},
\end{gathered}
$$

satisfies

$$
\|\mathbf{w}\|_{\mathcal{C}((0, T), H)} \leqslant\|\mathbf{w}(0)\|_{2}+1,
$$

and, for two functions $\mathbf{v}_{1}^{*}, \mathbf{v}_{2}^{*}$ satisfying (26), the solutions of

$$
\begin{gathered}
\frac{\mathrm{d} \mathbf{w}_{i}}{\mathrm{~d} t}=F\left(\mathbf{w}_{i}, \mathbf{v}_{i}^{*}\right), \\
\mathbf{w}_{i}(0)=-\alpha \Delta \mathbf{B}_{0}+\mathbf{B}_{0},
\end{gathered}
$$

satisfy

$$
\left\|\mathbf{w}_{1}-\mathbf{w}_{2}\right\|_{\mathcal{C}((0, T), H)} \leqslant \frac{1}{2}\left\|\mathbf{v}_{1}^{*}-\mathbf{v}_{2}^{*}\right\|_{L^{2}((0, T), V)} .
$$

(b) For $\mathbf{w}^{*} \in \mathcal{C}((0, T), H)$, with

$$
\left\|\mathbf{w}^{*}\right\|_{\mathcal{C}((0, T), H)} \leqslant\|\mathbf{w}(0)\|_{2}+1
$$

the solution of

$$
\begin{gathered}
\frac{\partial \mathbf{v}}{\partial t}=\nu \Delta \mathbf{v}-\mathbf{v} \cdot \nabla \mathbf{v}+G\left(\mathbf{w}^{*}\right)-\nabla p+\mathbf{f}, \\
\mathbf{v}(0)=\mathbf{v}_{0}
\end{gathered}
$$

satisfies

$$
\|\mathbf{v}\|_{L^{2}((0, T), V)} \leqslant \frac{k_{p}}{\sqrt{\nu}}\left\|\mathbf{v}_{0}\right\|_{H^{1}}+1,
$$

and, for two functions $\mathbf{w}_{1}^{*}, \mathbf{w}_{2}^{*}$ satisfying (33), the solutions of (34) with parameters $\mathbf{w}_{1}^{*}$ and $\mathbf{w}_{2}^{*}$ satisfy 


$$
\left\|\mathbf{v}_{1}-\mathbf{v}_{2}\right\|_{L^{2}((0, T), V)} \leqslant \frac{1}{2}\left\|\mathbf{w}_{1}^{*}-\mathbf{w}_{2}^{*}\right\|_{\mathcal{C}((0, T), H)}
$$

That $T$ may be found satisfying (a) follows from Theorems 2.3 and 2.4, whereas proposition 3.1 and Theorem 3.2 guarantee that for $T$ small enough, it also satisfies (b). Then the mapping defined in the product

$$
\bar{B}_{L^{2}((0, T), V)}\left(\frac{k_{p}}{\sqrt{\nu}}\left\|\mathbf{v}_{0}\right\|_{H^{1}}+1\right) \times \bar{B}_{\mathcal{C}((0, T), H)}\left(\left\|\mathbf{w}_{0}\right\|_{2}+1\right)
$$

and given by $\left(\mathbf{v}^{*}, \mathbf{w}^{*}\right) \rightarrow(\mathbf{v}, \mathbf{w})$, takes this product of balls into itself in a contractive manner. Therefore it has a unique fixed point. This is a solution of the equation, and conversely every solution is a fixed point, which guarantees uniqueness. Although in principle we only know that $\mathbf{v} \in L^{2}((0, T), V)$, since $\mathbf{w}$ and therefore $G(\mathbf{w})$ are uniformly bounded in $H$ for all $t \in(0, T)$, implies that $\mathbf{v}$, as the solution to a Navier-Stokes equation with a bounded forcing, belongs to $\mathcal{C}((0, T), V) \cap L^{2}((0, T), D(A))$. On the other hand, since $\|\mathbf{B}\|_{H^{2}} \leqslant c\|\mathbf{w}\|_{2}$, we find $\mathbf{B}$ $\in \mathcal{C}((0, T), D(A))$.

\section{FINAL CONSIDERATIONS}

The key difference with classical MHD is that the induction equation is actually simpler in our case, since the advective and diffusive terms are actually continuous functions of the new variable $\mathbf{w}=\alpha \boldsymbol{\nabla} \times \mathbf{J}+\mathbf{B}$. However, this will not help us to prove global existence. For one thing, the induction equation remains nonlinear in $\mathbf{w}$; and second, no matter how regular the magnetic field is, the momentum equation is a Navier-Stokes one, where global existence of solutions is a celebrated unsolved question. Still, it is apparent from our proofs that the difussive term $\eta \Delta \mathbf{B}$ does not play any role and can be dispensed with. This is in sharp contrast with one-fluid MHD, and it is a welcome feature, since most of the reconnection models where two-fluid MHD becomes necessary are actually infinitely conducting, i.e., the resistivity is taken as zero. That the two-fluid MHD equations yield more regularity than the classical MHD ones is apparent from the following energy inequality: it does not involve the $L^{2}$-norm of $\mathbf{w}$, whose evolution is complex, but rather the current density norm.

Theorem 5.1: Let $k_{p}$ be the Poincaré constant $\|\mathbf{v}\|_{2}^{2} \leqslant k_{p}\|\nabla \mathbf{v}\|_{2}^{2}$. The solutions to systems (4) and (5) satisfy, for as long as they are defined,

$$
\begin{aligned}
& \alpha\|\mathbf{J}(t)\|_{2}^{2}+\|\mathbf{B}(t)\|_{2}^{2}+\|\mathbf{v}(t)\|_{2}^{2}+2 \eta \int_{0}^{t}\|\nabla \mathbf{B}\|_{2}^{2}+\nu \int_{0}^{t}\|\nabla \mathbf{v}\|_{2}^{2} \mathrm{~d} s \\
& \quad \leqslant \alpha\|\mathbf{J}(0)\|_{2}^{2}+\|\mathbf{B}(0)\|_{2}^{2}+\|\mathbf{v}(0)\|_{2}^{2}+\frac{k_{p}}{\nu} \int_{0}^{t}\|\mathbf{f}\|_{2}^{2} \mathrm{~d} s .
\end{aligned}
$$

Proof: With our boundary conditions

$$
\int_{\Omega} J^{2} \mathrm{~d} V=\int_{\Omega} \mathbf{J} \cdot(\nabla \times \mathbf{B}) \mathrm{d} V=\int_{\Omega}(\nabla \times \mathbf{J}) \cdot \mathbf{B} \mathrm{d} V=-\int_{\Omega} \Delta \mathbf{B} \cdot \mathbf{B} \mathrm{d} V=\int_{\Omega}|\nabla \mathbf{B}|^{2} \mathrm{~d} V .
$$

Therefore

$$
\frac{\partial}{\partial t} \int_{\Omega} J^{2} \mathrm{~d} V=2 \int_{\Omega} \frac{\partial \nabla \mathbf{B}}{\partial t} \cdot \nabla \mathbf{B} \mathrm{d} V=-2 \int_{\Omega} \frac{\partial \Delta \mathbf{B}}{\partial t} \cdot \mathbf{B} \mathrm{d} V=2 \int_{\Omega} \frac{\partial(\nabla \times \mathbf{J})}{\partial t} \cdot \mathbf{B} \mathrm{d} V
$$

Let us multiply the momentum equation by $\mathbf{v}$, the induction one by $\mathbf{B}$, and add both. As in classical MHD, the advective terms add to zero, as well as 


$$
\int_{\Omega} \nabla\left(\frac{1}{2} B^{2}+p\right) \cdot \mathbf{v} \mathrm{d} V=\int_{\partial \Omega}\left(\frac{1}{2} B^{2}+p\right) \mathbf{v} \cdot \mathbf{n} \mathrm{d} \sigma=0 .
$$

As for the Hall term,

$$
\int_{\Omega}(\nabla \times(\mathbf{J} \times \mathbf{B})) \cdot \mathbf{B} \mathrm{d} V=\int_{\Omega}(\mathbf{J} \times \mathbf{B}) \cdot \mathbf{J} \mathrm{d} V=0 .
$$

Writing as usual

$$
\left|\int_{\Omega} \mathbf{f} \cdot \mathbf{v} \mathrm{d} V\right| \leqslant \sqrt{k_{p}}\|\mathbf{f}\|_{2}\|\nabla \mathbf{v}\|_{2} \leqslant \frac{\nu}{2}\|\nabla \mathbf{v}\|_{2}^{2}+\frac{k_{p}}{2 \nu}\|\mathbf{f}\|_{2}^{2},
$$

we obtain the stated inequality.

Thus, although the main existence theorem proved a finite-time bound for the maximum of $\|\mathbf{v}\|_{H^{1}}$ and $\|\mathbf{B}\|_{H^{2}}$, the present one remains valid for as long as the integrals make sense and is therefore more robust than the previous one. It plays the role of the energy inequality for MHD, but supplies more information about the smoothness of the field. Whereas in one-fluid MHD it was the total (kinetic plus magnetic) energy $\|\mathbf{v}\|_{2}^{2}+\|\mathbf{B}\|_{2}^{2}$ which remained bounded, here also the current density energy $\|\mathbf{J}\|_{2}^{2}$ does: hence the $H^{1}$-norm of the magnetic field is kept by the flow, which precludes any sharp gradients.

${ }^{1}$ N. A. Krall and A. W. Trivelpiece, Principles of Plasma Physics (McGraw-Hill, New York, 1973).

${ }^{2}$ V. M. Vasyliunas, Rev. Geophys. Space Phys. 13, 303 (1975).

${ }^{3}$ F. F. Chen, Introduction to Plasma Physics and Controlled Fusion (Plenum, New York, 1983).

${ }^{4}$ R. Temam, Infinite-Dimensional Dynamical Systems in Mechanics and Physics (Springer, New York, 1988).

${ }^{5}$ D. Biskamp, Nonlinear Magnetohydrodynamics (Cambridge University Press, Cambridge, UK, 1993).

${ }^{6}$ E. R. Priest and T. G. Forbes, Magnetic Reconnection: MHD Theory and Applications (Cambridge University Press, Cambridge, UK, 2000).

${ }^{7}$ D. Biskamp, Magnetic Reconnection in Plasmas (Cambridge University Press, Cambridge, UK, 2000).

${ }^{8}$ R. Temam, Navier-Stokes Equations and Nonlinear Functional Analysis, CBMS-NSF Regional Conference Series in Applied Mathematics (SIAM, Philadephia, 1995). 